\title{
N-methylthiomethylation of benzimidazoles with DMSO and their chemoselective oxidation to sulfoxides with $\mathrm{NaBiO}_{3}$
}

\author{
Chhanda Mukhopadhyay, ${ }^{* a}$ Pradip Kumar Tapaswi ${ }^{a}$, Swarbhanu Sarkar, ${ }^{\text {a }}$ \\ and Michael G. B. Drew \\ ${ }^{a}$ Department of Chemistry, University of Calcutta, 92 APC Road, Kolkata-700009, India \\ ${ }^{b}$ Department of Chemistry, The University, Whiteknights, Reading RG 6 6AD, UK \\ E-mail: cmukhop@yahoo.co.in
}

\begin{abstract}
A straightforward one-step method for the N-methylthiomethylation of benzimidazoles has been developed employing DMSO as a solvent and as a reagent. This methodology has been applied for the synthesis of diverse $\mathrm{N}$-methylthiomethyl derivatives of benzimidazoles. The products can be chemoselectively oxidized to the corresponding sulfoxides with $\mathrm{NaBiO}_{3}$ in acetic acid. Both the N-methylthiomethyl derivatives of benzimidazoles and their corresponding sulfoxides are important medicinal scaffolds.
\end{abstract}

Keywords: $N$-Methylthiomethylation of benzimidazoles, oxidation, sulfoxides, $\mathrm{DMSO}, \mathrm{NaBiO}_{3}$

\section{Introduction}

The imidazole nucleus is a privileged structural sub-unit encountered in numerous biologically important molecules ranging from natural products to pharmaceuticals. ${ }^{1}$ Several imidazole derivatives are widely used as organocatalysts, ${ }^{2}$ ionic liquids ${ }^{3}$ and $\mathrm{N}$-heterocyclic carbenes. ${ }^{4}$ Consequently, it is no wonder that the development of strategies for their synthesis and functionalization has instigated a growing interest in academia and in industry thereby continuing to be a significant subject in organic synthesis and medicinal chemistry. ${ }^{1}$

Both Burdon et al. ${ }^{5 \mathrm{a}}$ and Pfitzner et al. ${ }^{5 \mathrm{~b}}$ found almost simultaneously that phenols can be ortho-methylthiomethylated by dimethylsulfoxide (DMSO) in the presence of dicyclohexylcarbodiimide (DCC) and a proton source. Later, Hayashi and Oda found that the same reaction occurred using acetic anhydride instead of DCC at room temperature. ${ }^{5 \mathrm{c}}$ Gassman and Amick achieved the ortho-methylthiomethylation of phenols by the reaction of phenol and dimethylsulfide in presence of $\mathrm{N}$-chlorosuccinimide (NCS) and triethylamine. ${ }^{6 a}$ Foote et al. reported the ortho-methylthiomethylation of anilines by the reaction of anilines and dimethylsulfide in presence of tert-butyl hypochlorite and sodium methoxide. ${ }^{6 \mathrm{~b}}$ 
As several $\mathrm{N}$-methylthiomethyl derivatives of imidazoles are endowed with useful antiinflammatory, analgesic and antipyretic properties, ${ }^{7}$ we were very keen to develop similar type of reaction with imidazoles. The previous methods ${ }^{7,8}$ for the synthesis of $N$-methylthiomethyl imidazoles include a two step method: first, the formation of trialkylsilyl derivatives of imidazoles followed by reaction with DMSO at elevated temperature. This method has some serious drawbacks like two-step reaction procedure, drastic reaction condition and complex reaction methodology. Moreover, these procedures have not been generalized to different types of benzimidazole derivatives. We therefore wanted to develop a methodology that overcomes the earlier drawbacks.

The ever-growing interest and application of sulfoxides has attracted a growing interest to develop new methodologies for sulfoxide synthesis. Organic sulfoxides are valuable intermediates for the synthesis of fine chemicals and biologically active compounds. ${ }^{9}$

\section{Results and Discussions}

Considering the growing interest both in the conversion of imidazoles to $N$-methylthiomethyl imidazoles and in chemoselective oxidation of sulfides to sulfoxides, we, herein for the first time, report a straightforward conversion of several benzimidazoles to their N-methylthiomethyl counterparts followed by a mild, novel protocol for the chemoselective oxidation of sulfide to sulfoxide derivatives.

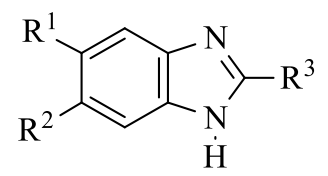

1
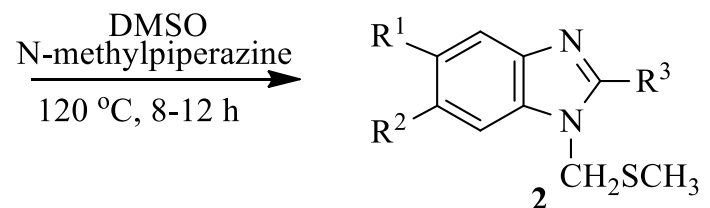

Scheme 1. N-Methylthiomethylation of benzimidazoles.

Initially, the reaction between 2-methylbenzimidazole and DMSO was selected as a model reaction to investigate the best reaction condition. After extensive studies with several bases, it was found that using $N$-methylpiperazine as a base at a moderate temperature produced the best yield of product after eight hours (Table 1, entry 8). The complete optimization table is given below. 
Table 1. Optimization for the N-methylthiomethylation of 2-methylbenzimidazole

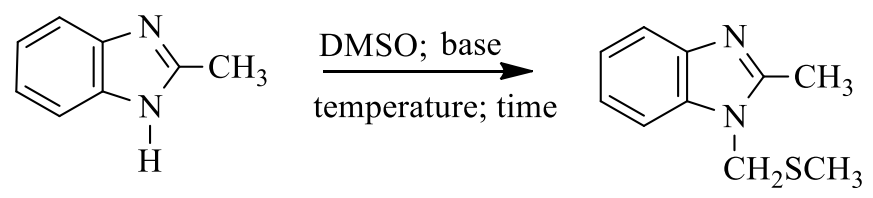

\begin{tabular}{ccccc}
\hline Entry & Base (equv.) & $\begin{array}{c}\text { Temperature } \\
\left({ }^{\circ} \mathrm{C}\right)\end{array}$ & Time (h) & $\begin{array}{c}\text { Yields (\%) } \\
\text { (isolated) }\end{array}$ \\
\hline 1 & $\square$ & 160 & 24 & 20 \\
2 & $\mathrm{Et}_{3} \mathrm{~N}(2)$ & 90 & 24 & 25 \\
3 & $\mathrm{~K}_{2} \mathrm{CO}_{3}(2)$ & 160 & 24 & 35 \\
4 & $\mathrm{Cs}_{2} \mathrm{CO}_{3}(2)$ & 160 & 24 & 35 \\
5 & $\mathrm{DABCO}(2)$ & 160 & 24 & 40 \\
6 & $\mathrm{NaH}(2)$ & 160 & 24 & 25 \\
7 & Piperidine (2) & 110 & 24 & 40 \\
8 & $\mathrm{NMP}(2)$ & 120 & 8 & 65 \\
9 & $\mathrm{NMP}(3)$ & 120 & 8 & 65 \\
10 & $\mathrm{NMP}(1.5)$ & 120 & 8 & 50 \\
11 & $\mathrm{NMP}(2)$ & 120 & 12 & 65 \\
12 & $\mathrm{NMP}(2)$ & 160 & 12 & 65 \\
\hline
\end{tabular}

Having established the optimized reaction conditions, imidazole and a wide variety of 2substituted benzimidazoles were investigated (Table 2) under the same reaction conditions. As a result, a broad spectrum of $\mathrm{N}$-methylthiomethylbenzimidazole(s) was obtained as shown in Table 2 . 
Table 2. N-Methylthiomethylation of benzimidazoles

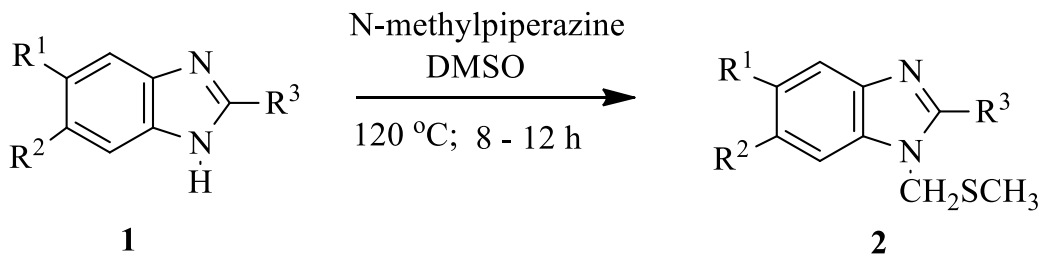

\begin{tabular}{|c|c|c|c|c|c|}
\hline Entry & $\begin{array}{c}\text { Product } \\
\text { Nos. }\end{array}$ & Products & $\begin{array}{l}\text { Time } \\
\text { (h) }\end{array}$ & $\begin{array}{c}\text { Yields } \\
(\%) \\
\text { (isolated) }\end{array}$ & References \\
\hline 1 & $\mathbf{2 a}$ & & 8 & 65 & 8 \\
\hline 2 & $2 b$ & & 10 & 59 & 8 \\
\hline 3 & $2 c$ & & 10 & 62 & 8 \\
\hline 4 & $2 d$ & & 8 & 60 & 8 \\
\hline 5 & $2 e$ & & 8 & 62 & - \\
\hline 6 & $2 f$ & & 8 & 66 & - \\
\hline 7 & $2 \mathrm{~g}$ & & 8.5 & 60 & - \\
\hline 8 & $2 h$ & & 8.5 & 60 & - \\
\hline 9 & $2 \mathrm{i}$ & & 8 & 65 & - \\
\hline 10 & $2 \mathbf{j}$ & & 10 & 65 & - \\
\hline
\end{tabular}


Table 2. Continued

\begin{tabular}{|c|c|c|c|c|c|}
\hline Entry & $\begin{array}{c}\text { Product } \\
\text { Nos. }\end{array}$ & Products & $\begin{array}{l}\text { Time } \\
\text { (h) }\end{array}$ & $\begin{array}{c}\text { Yields } \\
(\%) \\
\text { (isolated) }\end{array}$ & References \\
\hline 11 & $2 \mathrm{k}$ & & 12 & 58 & - \\
\hline 12 & 21 & & 8 & 64 & - \\
\hline 13 & $2 \mathrm{~m}$ & & 12 & 58 & - \\
\hline 14 & $2 n$ & & 11 & 58 & - \\
\hline 15 & 20 & & 10 & 60 & - \\
\hline 16 & $2 p$ & & 8 & 65 & - \\
\hline 17 & $2 q$ & & 8 & 65 & - \\
\hline 18 & $2 r$ & & 8 & 63 & - \\
\hline 19 & $2 s$ & $\mathrm{CH}_{2} \mathrm{SCH}_{3}$ & 12 & 30 & - \\
\hline
\end{tabular}

A base catalysed mechanism for the $\mathrm{N}$-methylthiomethylation of imidazole has been suggested below in which the valency of sulfur reduced from 4 (in DMSO) to 2 in the product. 


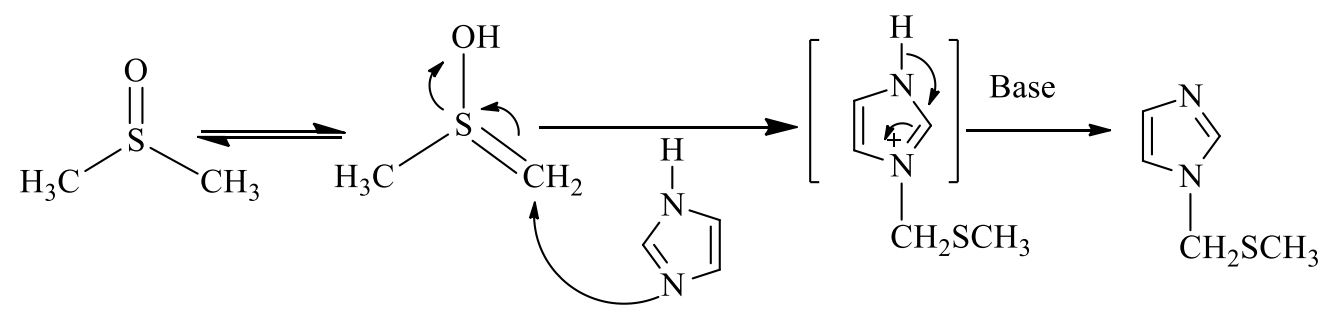

Scheme 2. Probable mechanism for the base catalysed N-methylthiomethylation of imidazole.

As illustrative example, the structure of $\mathrm{N}$-methylthiomethylation product of 2-phenyl benzimidazole (Table 2, entry 7) was assessed by X-ray crystallography and is given below in Figure 1.

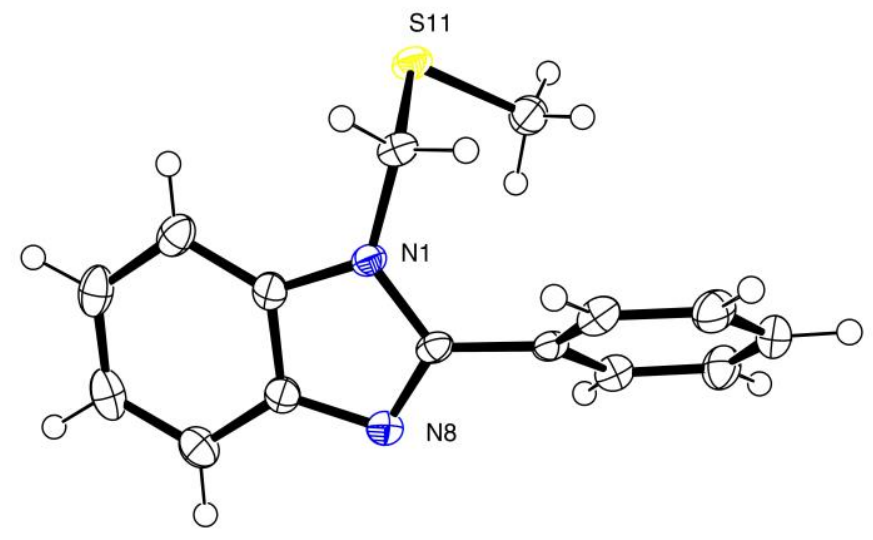

Figure 1. ORTEP diagram of product $2 \mathrm{~g}$ in (Table 2, entry 7 ) with ellipsoids at $50 \%$ probability (CCDC 780651).

The straightforward method for the synthesis of sulfoxides is the chemoselective oxidation of corresponding sulfide to sulfoxide. Therefore, once the $\mathrm{N}$-methylthiomethylation of benzimidazoles is achieved, further chemoselective oxidation of these sulfides to sulfoxides came into our mind immediately. There are a lot of reagents available for the oxidation of dialkyl, alkylaryl and diaryl sulfides to sulfoxides. ${ }^{10}$ Most of them cause over oxidation to sulfones. Therefore, the condition of the reaction, that is time, temperature and relative amounts of oxidants have to be controlled to avoid side product formation.

Recently, the oxidation of sulfides to sulfoxides by $\mathrm{H}_{2} \mathrm{O}_{2}$ has proved to be one of the most attractive methods. ${ }^{11}$ Very recently, Rostami and Akradi $^{12}$ reported boric acid as a highly efficient and eco-friendly catalyst for the selective oxidation of sulfide to sulfoxide at room temperature under solvent-free condition using $30 \% \mathrm{H}_{2} \mathrm{O}_{2}$ as an oxidant. We thought to test the oxidation of our sulfides under the same reaction condition. But unfortunately, $24 \mathrm{~h}$ of stirring at room temperature with 1.2 equivalent of $\mathrm{H}_{2} \mathrm{O}_{2}(30 \%), 0.1 \mathrm{mmol}$ of boric acid using $1 \mathrm{mmol}$ of our sulfide (Table 3, entry 1) only 5\% of sulfide was transformed into the desired product. Even 
after $24 \mathrm{~h}$ of heating under reflux condition using the same proportion of $\mathrm{H}_{2} \mathrm{O}_{2}$, boric acid and sulfide, only $40 \%$ transformation of the sulfide to the desired sulfoxides has been achieved. This unsatisfactory result forced us to search for a better oxidizing agent for the chemoselective oxidation of our sulfides to sulfoxides. After a thorough screening with the common oxidizing agents, $\mathrm{NaBiO}_{3}$ in acetic acid came out as the best choice for chemoselective oxidation of our sulfides to sulfoxides (Scheme 3). The presence of acetic acid is extremely important as the reaction failed to produce the desired product in its absence. It has also noticed that small amounts of acetone as co-solvent increases the yield of the reaction. ${ }^{13}$ Using this methodology a completely new class of organic sulfoxides has been prepared (Scheme 3, Table 3)<smiles>[R]c1cc2nc([R])n(CC)c2cc1[R]</smiles>

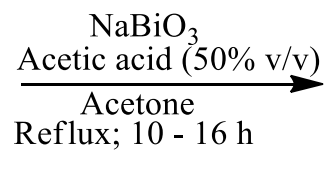

5

6 3f
10

80

10

82

${ }_{\mathrm{CH}} \mathrm{S}(=\mathrm{O}) \mathrm{CH}_{3}$

12

12

80<smiles>CO[Si]=O</smiles>

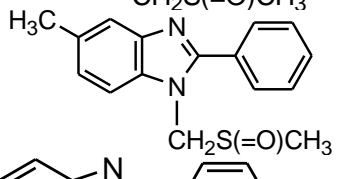

3e

14

16<smiles>CO[SiH2]OC</smiles>

Scheme 3. Chemoselective oxidation of sulfides to sulfoxides.

\begin{tabular}{|c|c|c|c|c|}
\hline Entry & $\begin{array}{c}\text { Product } \\
\text { Nos. }\end{array}$ & Products & Time $(\mathrm{h})$ & $\begin{array}{c}\text { Yields (\%) } \\
\text { (isolated) }\end{array}$ \\
\hline 1 & $\mathbf{3 a}$ & & 12 & 82 \\
\hline 2 & $3 b$ & & 12 & 80 \\
\hline 3 & $3 c$ & & 10 & 80 \\
\hline 4 & $3 d$ & & 10 & 82 \\
\hline 5 & $3 e$ & & 14 & 78 \\
\hline 6 & $3 f$ & & 16 & 70 \\
\hline
\end{tabular}

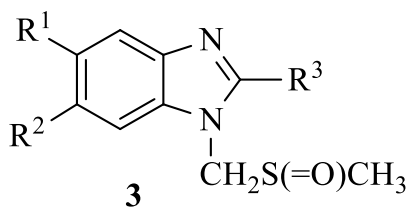

Table 3. Chemoselective oxidation of sulfides to sulfoxides by $\mathrm{NaBiO}_{3}$ in acetic acid

The mechanism of the oxidation reaction by $\mathrm{NaBiO}_{3}$ in acetic acid is not clear. Kon and McNeils predicted ${ }^{14}$ that the oxidation of phenol in acetic acid medium by $\mathrm{NaBiO}_{3}$ involved a 
two electron oxidation and considering this reference we may predict that the oxidation of sulfides to sulfoxides in our case may also proceed through 2-electron oxidation.

The final structure of the sulfoxide was confirmed by the X-ray crystal structure of the sulfoxide product 3c (Table 3, entry 3) which is given below in Figure 2.

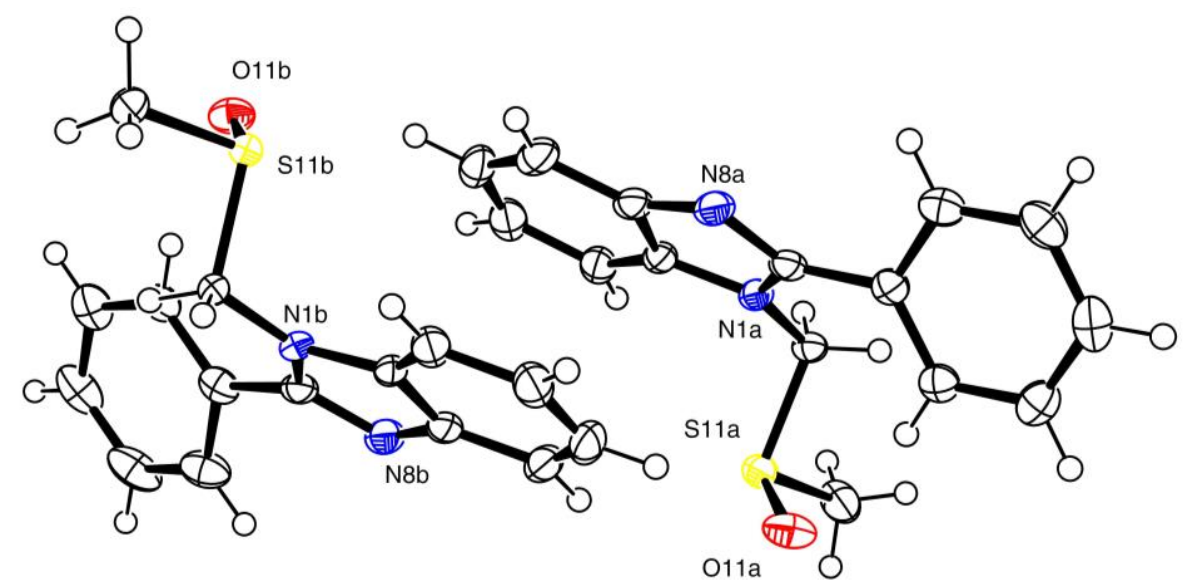

Figure 2. ORTEP diagram of product 3c (Table 3, entry 3) (CCDC 780781) showing both molecules in the asymmetric unit with ellipsoids at $30 \%$ probability. The solvent methanol and water molecules are not shown.

Figure 2 contains two molecules in the asymmetric unit together with five water molecules, three of which have reduced occupancy and one methanol, also with reduced occupancy. The two molecules in the asymmetric unit have similar geometries as is apparent from figure 2. In particular the angles around the N1-C and C-S11 bonds are 109.1(3) in A, -106.2(3) in B and 69.9(2) in A, 74.2(2) in B respectively thus showing that the molecules are opposite enantiomers. The phenyl rings in the two structures stack with the six C...C distances ranging from 3.54 to $3.75 \AA$. The angle between the two phenyl ring planes is $2.3(1)^{\circ}$. There is, therefore, definite evidence of pi-stacking in this molecule.

\section{Conclusion}

In conclusion, a straightforward method for $\mathrm{N}$-methylthiomethylation of benzimidazoles has been described under moderate reaction conditions. Relatively moderate reaction condition, a wide range of substrate affordability and high yields are the major advantages of this methodology. Further, chemoselective oxidation of these N-methylthiomethylbenzimidazoles to sulfoxides has been achieved using $\mathrm{NaBiO}_{3}$ in acetic acid medium. This is the first report of synthesis of this kind of sulfoxide which can be expected to open a new arena of research for synthetic organic chemists. 


\section{Experimental Section}

General. All the chemicals were purchased from Aldrich Chemical Company and Spectrochem, Pvt. Ltd. (Mumbai, India). Silica Gel G with binder from Spectrochem, Pvt. Ltd. Mumbai, India was used for thin layer chromatography. ${ }^{1} \mathrm{H}$ and ${ }^{13} \mathrm{C}$ NMR spectra were obtained on Bruker 300 $\mathrm{MHz}$ instrument at 300 and $75 \mathrm{MHz}$ respectively. $\mathrm{CDCl}_{3}$ was purchased from Aldrich Chemical Company and $\mathrm{d}_{6}$-DMSO from CIL. Melting points were determined on an electrical melting point apparatus with an open capillary and are uncorrected. IR spectra were recorded on a Perkin Elmer Spectrophotometer RX / FT-IR system. The C-H-N analyses were carried out on a 2400series II CHNS Analyzer, Perkin Elmer (USA). Ion cyclotron resonance Fourier transform HRMS was performed on a Micromass ZQ instrument (Waters) (location: Indian Institute of Chemical Biology, 4, Raja S. C. Mullick Road, Kolkata-700032) and ESIMS of the compounds were recorded on a Waters LC-MS-MS (quattro micro mass) instrument (Chemgen Pharma International Pvt. Ltd., Dr. Siemens Street, Block GP, Sector-V, Salt Lake City, Kolkata700091).

Preparation of $\mathbf{N}$-methylthiomethylbenzimidazoles. In a round bottomed flask (10 $\mathrm{mL})$, benzimidazoles ( $1 \mathrm{mmol})$, N-methylpiperazine $(2 \mathrm{mmol})$ and dry DMSO $(2 \mathrm{~mL})$ were mixed and heated on oil bath for stipulated time (monitored by TLC). After the completion of the reaction, the solvent was removed under reduced pressure in a rotary evaporator. The concentrated crude product was separated by column chromatography using silica gel (60-120 mesh) and petroleum ether $\left(60-80{ }^{\circ} \mathrm{C}\right) /$ ethyl acetate as eluant. The characteristic data for all the new compounds are given below.

2,5-Dimethyl-1-methylsulphanylmethyl-1H-benzimidazole (2e, Table 2, entry 5). Mixture of two tautomers; colorless semisolid; ${ }^{1} \mathrm{H} \mathrm{NMR}\left(300 \mathrm{MHz}, \mathrm{CDCl}_{3}\right): \delta(1.86$ and 1.82) (two s, $3 \mathrm{H}$ ), (2.31 and 2.28) (two s, 3H), 2.43 (s, 3H), 4.89 (s, 2H), 6.89 (d, $J=8.1 \mathrm{~Hz}, 1 \mathrm{H}),(6.98$ and 7.30) (two s, 1H, tautomeric and aromatic), (7.07 and 7.38) (two $\mathrm{d}, \mathrm{J}=8.1 \mathrm{~Hz}, 1 \mathrm{H}$, tautomeric and aromatic); ${ }^{13} \mathrm{C} \mathrm{NMR}\left(75 \mathrm{MHz}, \mathrm{CDCl}_{3}\right): \delta 13.8,14.1,21.3,21.6,45.8,46.0,108.9,109.4,118.4$, 118.8, 123.6, 123.6, 131.8, 132.2, 132.9, 135.0, 140.1, 142.3, 150.8, 151.3; ESI-MS (m/z): 207.0 $\left(\mathrm{M}^{+}+1\right)$; IR (neat, $\left.\mathrm{cm}^{-1}\right)$ : 682, 852, 1090, 1276, 1380, 1463, 1532, 2376, $2909 \mathrm{~cm}^{-1}$; Anal. Calc. for $\mathrm{C}_{11} \mathrm{H}_{14} \mathrm{~N}_{2} \mathrm{~S}$ : C 64.04, H 6.84, N 13.58; Found: C 63.94, H 6.90, N 13.62\%.

1-Methylsulphanylmethyl-2,5,6-trimethyl-1H-benzimidazole (2f, Table 2, entry 6). Colorless solid; m.p: $112-114{ }^{\circ} \mathrm{C}$ (EtOAc); ${ }^{1} \mathrm{H}$ NMR (300 MHz, $\left.\mathrm{CDCl}_{3}\right): \delta 2.02$ (s, 3H), 2.33 (s, 3H), $2.36(\mathrm{~s}, 3 \mathrm{H}), 2.59(\mathrm{~s}, 3 \mathrm{H}), 5.05(\mathrm{~s}, 2 \mathrm{H}), 7.12(\mathrm{~s}, 1 \mathrm{H}), 7.42(\mathrm{~s}, 1 \mathrm{H}) ;{ }^{13} \mathrm{C} \mathrm{NMR}\left(75 \mathrm{MHz}, \mathrm{CDCl}_{3}\right): \delta$ 13.9, 14.1, 20.1, 20.4, 46.0, 109.8, 119.3, 130.9, 131.2, 133.4, 140.9, 150.5; ESI-MS (m/z): 221.1 $\left(\mathrm{M}^{+}+1\right)$; HRMS $(\mathrm{m} / \mathrm{z})$ for $\mathrm{C}_{12} \mathrm{H}_{16} \mathrm{~N}_{2} \mathrm{~S}$; calcd 220.1034, found 220.1032; IR $\left(\mathrm{KBr}, \mathrm{cm}^{-1}\right)$ : 675, 847, 990, 1291, 1389, 1453, 1521, 2372, $2919 \mathrm{~cm}^{-1}$; Anal. Calc. for $\mathrm{C}_{12} \mathrm{H}_{16} \mathrm{~N}_{2} \mathrm{~S}: \mathrm{C}$ 65.41, H 7.32, N 12.71; Found: C 65.29, H 7.40, N 12.75.

1-Methylsulphanylmethyl-2-phenyl-1H-benzimidazole (2g, Table 2, entry 7). Pale yellow solid; m.p: 156 - $158{ }^{\circ} \mathrm{C}$ (EtOAc); ${ }^{1} \mathrm{H}$ NMR (300 MHz, $\left.\mathrm{CDCl}_{3}\right): \delta 1.91(\mathrm{~s}, 3 \mathrm{H}), 5.24(\mathrm{~s}, 2 \mathrm{H})$, 
$7.34-7.28(\mathrm{~m}, 2 \mathrm{H}), 7.51-7.49(\mathrm{~m}, 4 \mathrm{H}), 7.86-7.74(\mathrm{~m}, 3 \mathrm{H}) ;{ }^{13} \mathrm{C} \mathrm{NMR}\left(75 \mathrm{MHz}, \mathrm{CDCl}_{3}\right): \delta$ 14.6, 47.3, 110.8, 119.9, 122.9, 123.0, 128.7, 129.5, 129.7, 129.9, 134.9, 142.7, 153.7; ESI-MS $(\mathrm{m} / \mathrm{z})$ : $255.0\left(\mathrm{M}^{+}+1\right)$; HRMS (m/z) for $\mathrm{C}_{15} \mathrm{H}_{14} \mathrm{~N}_{2} \mathrm{~S}$; calcd 254.0878, found 254.0876; IR $(\mathrm{KBr}$, $\mathrm{cm}^{-1}$ ): 750, 1000, 1077, 1151, 1277, 1365, 1447, 2374, 2920, 2993, $3047 \mathrm{~cm}^{-1}$; Anal. Calc. for $\mathrm{C}_{15} \mathrm{H}_{14} \mathrm{~N}_{2} \mathrm{~S}$ : C 70.83, H 5.55, N 11.01; Found: C 70.71, H 5.65, N 11.03.

5-Methyl-1-methylsulphanylmethyl-2-phenyl-1H-benzimidazole (2h, Table 2, entry 8). Mixture of two tautomers; yellow solid; m.p: 72 - $74{ }^{\circ} \mathrm{C}$ (EtOAc); ${ }^{1} \mathrm{H}$ NMR $\left(300 \mathrm{MHz}, \mathrm{CDCl}_{3}\right.$ ): $\delta 1.91(\mathrm{~s}, 3 \mathrm{H}),(2.52$ and 2.49) (two s, 3H), $5.24(\mathrm{~s}, 2 \mathrm{H}), 7.15(\mathrm{~d}, J=8.1 \mathrm{~Hz}, 1 \mathrm{H}),(7.31$ and 7.62) (two d, $J=0.6 \mathrm{~Hz}, 1 \mathrm{H}$, tautomeric and aromatic), (7.40 and 7.71) (two d, $J=8.2 \mathrm{~Hz}, 1 \mathrm{H}$, tautomeric and aromatic), $7.56-7.47(\mathrm{~m}, 3 \mathrm{H}), 7.82-7.52(\mathrm{~m}, 2 \mathrm{H}) ;{ }^{13} \mathrm{C} \mathrm{NMR}\left(75 \mathrm{MHz}, \mathrm{CDCl}_{3}\right)$ : $\delta 14.6,21.5,21.8,47.2,47.4,110.4,110.7,119.4,119.6,124.6,128.8,129.5,129.6,129.7$, 129.9, 128.0, 132.8, 133.0, 133.1, 135.1, 140.8, 142.9, 153.2, 153.6; ESI-MS (m/z): 269.2 $\left(\mathrm{M}^{+}+1\right)$; IR (KBr, cm $\left.{ }^{-1}\right): 701,780,800,1278,1382,1444,1620,2321,1922,3060 \mathrm{~cm}^{-1}$; Anal. Calc. for $\mathrm{C}_{16} \mathrm{H}_{16} \mathrm{~N}_{2} \mathrm{~S}$ : C 71.61, H 6.01, N 10.44; Found: C 71.54, H 6.05, N 10.47.

5,6-Dimethyl-1-methylsulphanylmethyl-2-phenyl-1H-benzimidazole (2i, Table 2, entry 9). White crystalline solid; m.p; $82-84{ }^{\circ} \mathrm{C}\left(\right.$ EtOAc); ${ }^{1} \mathrm{H}$ NMR $\left(300 \mathrm{MHz}, \mathrm{CDCl}_{3}\right): \delta 1.91(\mathrm{~s}, 3 \mathrm{H})$, 2.39 (s, 3H), $2.42(\mathrm{~s}, 3 \mathrm{H}), 5.24(\mathrm{~s}, 2 \mathrm{H}), 7.29(\mathrm{~s}, 1 \mathrm{H}), 7.52-7.49(\mathrm{~m}, 3 \mathrm{H}), 7.58(\mathrm{~s}, 1 \mathrm{H}), 7.76-$ $7.72(\mathrm{~m}, 2 \mathrm{H}) ;{ }^{13} \mathrm{C} \mathrm{NMR}\left(75 \mathrm{MHz}, \mathrm{CDCl}_{3}\right): \delta 14.5,20.2,20.6,47.3,111.0,120,128.7,129.5$, 129.7, 130.1, 131.8, 132.2, 133.5, 141.6, 153.1; ESI-MS (m/z): $283.1\left(\mathrm{M}^{+}+1\right)$; HRMS (m/z) for $\mathrm{C}_{17} \mathrm{H}_{18} \mathrm{~N}_{2} \mathrm{~S}$; calcd 282.1191, found 282.1201; IR $\left(\mathrm{KBr}, \mathrm{cm}^{-1}\right)$ : 701, 847, 999, 1072, 1177, 1280, 1383, 1461, 1627, 2330, $2923 \mathrm{~cm}^{-1}$; Anal. Calc. for $\mathrm{C}_{17} \mathrm{H}_{18} \mathrm{~N}_{2} \mathrm{~S}$ : C 72.30, H 6.42, N 9.92; Found: C 72.19, H 6.49, N 9.96.

2-(4'-Chlorophenyl)-1-methylsulphanylmethyl-1H-benzimidazole (2j, Table 2, entry 10 ). Colorless crystalline solid; m.p: $100-102{ }^{\circ} \mathrm{C}$ (EtOAc); ${ }^{1} \mathrm{H}$ NMR $\left(300 \mathrm{MHz}, \mathrm{CDCl}_{3}\right): \delta 2.00(\mathrm{~s}$, $3 \mathrm{H}), 5.26(\mathrm{~s}, 2 \mathrm{H}), 7.38-7.32(\mathrm{~m}, 2 \mathrm{H}), 7.53(\mathrm{td}, J=6.6$ and $1.8 \mathrm{~Hz}, 3 \mathrm{H}), 7.77(\mathrm{td}, J=8.4$ and 2.0 $\mathrm{Hz}, 2 \mathrm{H}), 7.85-7.81(\mathrm{~m}, 1 \mathrm{H}) ;{ }^{13} \mathrm{C} \mathrm{NMR}\left(75 \mathrm{MHz}, \mathrm{CDCl}_{3}\right): \delta 14.9,47.4,110.7,120.1,123.1$, 123.3, 128.5, 129.2, 130.9, 135.3, 136.3, 143.0, 152.6; ESI-MS (m/z): $289.0\left(\mathrm{M}^{+}+1\right)$; HRMS $(\mathrm{m} / \mathrm{z})$ for $\mathrm{C}_{15} \mathrm{H}_{13} \mathrm{ClN}_{2} \mathrm{~S}$; calcd 288.0488, found 288.0482; IR (KBr, $\left.\mathrm{cm}^{-1}\right)$ : 740, 1049, 1089, 1182, 1242, 1346, 1529, 2374, 2866, 2922, $3090 \mathrm{~cm}^{-1}$ Anal. Calc. for $\mathrm{C}_{15} \mathrm{H}_{13} \mathrm{ClN}_{2} \mathrm{~S}$ : C 62.38, H 4.45, N 9.70; Found: C 62.22, H 4.55, N 9.76

1-Methylsulphanylmethyl-2-(4'-nitrophenyl)-1H-benzimidazole (2k, Table 2 , entry 11). Yellow solid; m.p: $164-166{ }^{\circ} \mathrm{C}\left(\right.$ EtOAc); ${ }^{1} \mathrm{H}$ NMR $\left(300 \mathrm{MHz}, \mathrm{CDCl}_{3}\right): \delta 2.04(\mathrm{~s}, 3 \mathrm{H}), 5.33(\mathrm{~s}$, $2 \mathrm{H}), 7.47-7.38(\mathrm{~m}, 2 \mathrm{H}), 7.62-7.58(\mathrm{~m}, 1 \mathrm{H}), 7.90-7.86(\mathrm{~m}, 1 \mathrm{H}), 8.12(\mathrm{~d}, J=8.4 \mathrm{~Hz}, 2 \mathrm{H}), 8.41$ $(\mathrm{d}, J=8.4 \mathrm{~Hz}, 2 \mathrm{H}) ;{ }^{13} \mathrm{C} \mathrm{NMR}\left(75 \mathrm{MHz}, \mathrm{CDCl}_{3}\right): \delta 15.1,48.0,111.1,119.9,124.2,124.2,124.6$, 130.8, 134.8, 135.1, 141.4, 148.9, 150.5; ESI-MS (m/z): $300.0\left(\mathrm{M}^{+}+1\right) ; \operatorname{HRMS}(\mathrm{m} / \mathrm{z})$ for $\mathrm{C}_{15} \mathrm{H}_{13} \mathrm{~N}_{3} \mathrm{O}_{2} \mathrm{~S}$; calcd 299.0728, found 299.0726; IR (KBr, $\left.\mathrm{cm}^{-1}\right)$ : 746, 855, 1281, 1340, 1452 , 1521, 2210, $2923 \mathrm{~cm}^{-1}$; Anal. Calc. for $\mathrm{C}_{15} \mathrm{H}_{13} \mathrm{~N}_{3} \mathrm{O}_{2} \mathrm{~S}$ : C 60.18, H 4.38, N 14.04; Found: C 60.10, H 4.46, N 14.04.

2-(4'-N,N-dimethylaminophenyl)-1-methylsulphanylmethyl-1H-benzimidazole (2l, Table 2, entry 12). Colorless crystalline solid; m.p: $150-152{ }^{\circ} \mathrm{C}(\mathrm{EtOAc}) ;{ }^{1} \mathrm{H} \mathrm{NMR}\left(300 \mathrm{MHz}, \mathrm{CDCl}_{3}\right)$ : 
$\delta 1.98(\mathrm{~s}, 3 \mathrm{H}), 3.03(\mathrm{~s}, 6 \mathrm{H}), 5.29(\mathrm{~s}, 2 \mathrm{H}), 6.79(\mathrm{~d}, J=9.0 \mathrm{~Hz}, 2 \mathrm{H}), 7.32-7.26(\mathrm{~m}, 2 \mathrm{H}), 7.51-$ $7.46(\mathrm{~m}, 1 \mathrm{H}), 7.70(\mathrm{~d}, J=9.0 \mathrm{~Hz}, 2 \mathrm{H}), 7.84-7.79(\mathrm{~m}, 1 \mathrm{H}) ;{ }^{13} \mathrm{C} \mathrm{NMR}\left(75 \mathrm{MHz}, \mathrm{CDCl}_{3}\right): \delta 14.7$, 40.1, 47.6, 110.6, 111.8, 115.8, 119.1, 122.6, 122.9, 130.6, 134.8, 141.9, 151.4, 154.4; ESI-MS (m/z): $298.0\left(\mathrm{M}^{+}+1\right)$; HRMS (m/z) for $\mathrm{C}_{17} \mathrm{H}_{19} \mathrm{~N}_{3} \mathrm{~S}$; calcd 297.1300, found 297.1290; IR (KBr, $\left.\mathrm{cm}^{-1}\right):$ 743, 816, 940, 1062, 1185, 1277, 1367, 1483, 1608, 2376, $2918 \mathrm{~cm}^{-1}$; Anal. Calc. for $\mathrm{C}_{17} \mathrm{H}_{19} \mathrm{~N}_{3} \mathrm{~S}: \mathrm{C}$ 68.65, H 6.44, N 14.13; Found: C 68.57, H 6.46, N 14.19.

2-(3',4'-Dimethoxyphenyl)-1-methylsulphanylmethyl-1H-benzimidazole $(\mathbf{2 m}$, Table 2 , entry 13). Pale yellow solid; m.p: $94-96{ }^{\circ} \mathrm{C}$ (EtOAc); ${ }^{1} \mathrm{H}$ NMR $\left(300 \mathrm{MHz}, \mathrm{CDCl}_{3}\right): \delta 1.98(\mathrm{~s}, 3 \mathrm{H})$, $3.89(\mathrm{~s}, 3 \mathrm{H}), 3.91(\mathrm{~s}, 3 \mathrm{H}), 5.28(\mathrm{~s}, 2 \mathrm{H}), 6.93(\mathrm{~d}, J=8.1 \mathrm{~Hz}, 1 \mathrm{H}), 7.30-7.25(\mathrm{~m}, 2 \mathrm{H}), 7.35(\mathrm{~d}, J=$ $8.6 \mathrm{~Hz}, 1 \mathrm{H}), 7.41(\mathrm{~s}, 1 \mathrm{H}), 7.50-7.46(\mathrm{~m}, 1 \mathrm{H}), 7.78-7.75(\mathrm{~m}, 1 \mathrm{H}) ;{ }^{13} \mathrm{C} \mathrm{NMR}\left(75 \mathrm{MHz}, \mathrm{CDCl}_{3}\right)$ : $\delta 15.0,47.9,56.0,56.3,110.9,111.2,112.8,119.0,120.4,122.6,123.6,123.7,134.5,140.3$, 149.4, 151.1, 153.0; ESI-MS (m/z): $315.1\left(\mathrm{M}^{+}+1\right)$; IR (KBr, $\left.\mathrm{cm}^{-1}\right): 745,1021,1142,1260,1451$, 1605, 2374, $2925 \mathrm{~cm}^{-1}$; Anal. Calc. for $\mathrm{C}_{17} \mathrm{H}_{18} \mathrm{~N}_{2} \mathrm{O}_{2} \mathrm{~S}$ : C 64.94, H 5.77 N 8.91; Found: C 64.83, H 5.85, N 8.94.

2-(3',4'-Dimethoxyphenyl)-5,6-dimethyl-1-methylsulphanylmethyl-1H-benzimidazole (2n, Table 2, entry 14). Pale yellow solid; m.p: 96- $98{ }^{\circ} \mathrm{C}$ (EtOAc); ${ }^{1} \mathrm{H}$ NMR (300 MHz, $\left.\mathrm{CDCl}_{3}\right): \delta$ 2.02 (s, 3H), 2.38 (s, 3H), 2.42 (s, 3H), 3.90 (s, 3H), 3.94 (s, 3H), 5.24 (s, 2H), 6.98 (d, J = 8.2 $\mathrm{Hz}, 1 \mathrm{H}), 7.26(\mathrm{~s}, 1 \mathrm{H}), 7.33$ (dd, $J=8.2$ and $1.8 \mathrm{~Hz}, 1 \mathrm{H}), 7.38(\mathrm{~d}, J=2.4 \mathrm{~Hz}, 1 \mathrm{H}), 7.56(\mathrm{~s}, 1 \mathrm{H})$; ${ }^{13} \mathrm{C}$ NMR $\left(75 \mathrm{MHz} \mathrm{CDCl}_{3}\right): \delta 14.8,20.2,20.7,47.5,56.0,56.1,110.8,111.1,112.7,119.9$, 122.3, 122.6, 131.8, 132.2, 133.8, 141.5, 149.2, 150.4, 153.1; ESI-MS (m/z): $343.1\left(\mathrm{M}^{+}+1\right)$; IR $\left(\mathrm{KBr}, \mathrm{cm}^{-1}\right)$ : 1020, 1141, 1261, 1379, 1458, 1602, 2372, 2853, $2924 \mathrm{~cm}^{-1}$; Anal. Calc. for $\mathrm{C}_{19} \mathrm{H}_{22} \mathrm{~N}_{2} \mathrm{O}_{2} \mathrm{~S}$ : C 66.64, H 6.48, N 8.18; Found: C 66.54, H 6.55, N 8.21.

5,6-Dimethyl-2-(4'-methoxyphenyl)-1-methylsulphanylmethyl-1H-benzimidazole (2o, Table 2, entry 15). Pale yellow solid; m.p: $102-104{ }^{\circ} \mathrm{C}$ (EtOAc); ${ }^{1} \mathrm{H}$ NMR (300 MHz, CDCl $): \delta 1.94$ (s, 3H), 2.39 (s, 3H), $2.42(\mathrm{~s}, 3 \mathrm{H}), 3.88(\mathrm{~s}, 3 \mathrm{H}), 5.23(\mathrm{~s}, 2 \mathrm{H}), 7.03(\mathrm{td}, J=8.7$, and $2.3 \mathrm{~Hz}, 2 \mathrm{H})$, $7.27(\mathrm{~s}, 1 \mathrm{H}), 7.56(\mathrm{~s}, 1 \mathrm{H}), 7.72(\mathrm{td}, J=8.7$ and $2.3 \mathrm{~Hz}, 2 \mathrm{H}) ;{ }^{13} \mathrm{C} \mathrm{NMR}\left(75 \mathrm{MHz}, \mathrm{CDCl}_{3}\right): \delta 14.6$, 20.2, 20.6, 47.3, 55.4, 110.9, 114.2, 119.9, 122.4, 131.0, 131.8, 132.0, 133.6, 141.5, 153.1, 160.8; ESI-MS (m/z): $313.1\left(\mathrm{M}^{+}+1\right)$; IR (KBr, $\left.\mathrm{cm}^{-1}\right)$ : 847, 1030, 1175, 1243, 1457, 1606, 2850, $2923 \mathrm{~cm}^{-1}$; Anal. Calc. for $\mathrm{C}_{18} \mathrm{H}_{20} \mathrm{~N}_{2} \mathrm{OS}$ : C 69.20, H 6.45, N 8.97; Found: C 69.12, H 6.48, N 9.02 .

2-(4'-Chlorophenyl)-5,6-dimethyl-1-methylsulphanylmethyl-1H-benzimidazole (2p, Table 2, entry 16). Colourless crystalline solid; m.p: 178 - $180{ }^{\circ} \mathrm{C}$ (EtOAc); ${ }^{1} \mathrm{H}$ NMR (300 MHz, $\left.\mathrm{CDCl}_{3}\right): \delta 1.95(\mathrm{~s}, 3 \mathrm{H}), 2.38(\mathrm{~s}, 3 \mathrm{H}), 2.43(\mathrm{~s}, 3 \mathrm{H}), 5.26(\mathrm{~s}, 2 \mathrm{H}), 7.31(\mathrm{~s}, 1 \mathrm{H}), 7.50(\mathrm{~d}, J=8.4 \mathrm{~Hz}$, 2H), $7.57(\mathrm{~s}, 1 \mathrm{H}), 7.77(\mathrm{~d}, J=8.4 \mathrm{~Hz}, 2 \mathrm{H}) ;{ }^{13} \mathrm{C} \mathrm{NMR}\left(75 \mathrm{MHz}, \mathrm{CDCl}_{3}\right): \delta 14.7,20.2,20.7,42.8$, 111.2, 119.0, 126.7, 129.3, 130.5, 131.0, 132.9, 133.2, 133.5, 136.9. 150.7; ESI-MS (m/z): 317.0 $\left(\mathrm{M}^{+}+1\right)$; HRMS (m/z) for $\mathrm{C}_{17} \mathrm{H}_{17} \mathrm{ClN}_{2} \mathrm{~S}$; calcd 316.0801, found 316.0793; IR ( $\left.\mathrm{KBr}, \mathrm{cm}^{-1}\right)$ : 843, 1000, 1087, 1288, 1319, 1461, 2376, $2921 \mathrm{~cm}^{-1}$; Anal. Calc. for $\mathrm{C}_{17} \mathrm{H}_{17} \mathrm{ClN}_{2} \mathrm{~S}$ : C 64.44, H 5.41, N 8.84; Found: C 64.33, H 5.50, N 8.86.

2-(4'-Bromophenyl)-5,6-dimethyl-1-methylsulphanylmethyl-1H-benzimidazole (2q, Table 2, entry 17). Colourless crystalline solid; m.p: $182-184{ }^{\circ} \mathrm{C}$ (EtOAc); ${ }^{1} \mathrm{H}$ NMR (300 MHz, 
$\left.\mathrm{CDCl}_{3}\right): \delta 1.95(\mathrm{~s}, 3 \mathrm{H}), 2.38(\mathrm{~s}, 3 \mathrm{H}), 2.42(\mathrm{~s}, 3 \mathrm{H}), 5.22(\mathrm{~s}, 2 \mathrm{H}), 7.29(\mathrm{~s}, 1 \mathrm{H}), 7.56(\mathrm{~s}, 1 \mathrm{H}), 7.70-$ $7.63(\mathrm{~m}, 4 \mathrm{H}) ;{ }^{13} \mathrm{C} \mathrm{NMR}\left(75 \mathrm{MHz}, \mathrm{CDCl}_{3}\right): \delta 14.8,20.2,20.7,47.8,111.3,119.0,125.3,127.1$, 131.1, 132.3, 132.9, 133.3, 133.6, 150.8; ESI-MS (m/z): $361.0\left(\mathrm{M}^{+}+1\right)$; HRMS $(\mathrm{m} / \mathrm{z})$ for $\mathrm{C}_{17} \mathrm{H}_{17} \mathrm{BrN}_{2} \mathrm{~S}$ : calcd 360.0296, found 360.0302; IR $\left(\mathrm{KBr}, \mathrm{cm}^{-1}\right)$ : 840, 1000, 1067, 1286, 1400, 1463, 1595, 2464, 2858, $2922 \mathrm{~cm}^{-1}$; Anal. Calc. for $\mathrm{C}_{17} \mathrm{H}_{17} \mathrm{BrN}_{2} \mathrm{~S}$ : C 56.51, H 4.74, N 7.75; Found: C 56.39, H 4.83, N 7.78.

\section{5,6-Dimethyl-2-(4'-N,N-dimethylaminophenyl)-1-methylsulphanylmethyl-1H-}

benzimidazole (2r, Table 2, entry 18). Pale yellow solid; m.p: $156-158{ }^{\circ} \mathrm{C}$ (EtOAc); ${ }^{1} \mathrm{H}$ NMR (300 MHz, $\left.\mathrm{CDCl}_{3}\right): \delta 1.96$ (s, 3H), 2.42 (s, 3H), 2.47 (s, 3H), 3.04 (s, 6H), 5.27 (s, 2H), 6.80 (d, $J=8.7 \mathrm{~Hz}, 2 \mathrm{H}), 7.26(\mathrm{~s}, 1 \mathrm{H}), 7.58(\mathrm{~s}, 1 \mathrm{H}), 7.68(\mathrm{~d}, J=9.0 \mathrm{~Hz}, 2 \mathrm{H}) ;{ }^{13} \mathrm{C} \mathrm{NMR}\left(75 \mathrm{MHz}, \mathrm{CDCl}_{3}\right)$ : $\delta 14.5,20.2,20.6,40.1,47.4,110.9,111.8,116.4,119.3,130.5,131.7,133.4,140.8,151.2$, 153.7; ESI-MS (m/z): $326.1\left(\mathrm{M}^{+}+1\right)$; IR $\left(\mathrm{KBr}, \mathrm{cm}^{-1}\right): 740,1049,1089,1182,1242,1346,1529$, 2374, 2866, 2922, $3090 \mathrm{~cm}^{-1}$; Anal. Calc. for $\mathrm{C}_{19} \mathrm{H}_{23} \mathrm{~N}_{3} \mathrm{~S}$ : C 70.11, H 7.12, N 12.91; Found: C 69.99, H 7.21, N 12.94.

2-(1H-Benzimidazol-2-yl)-1-methylsulphanylmethyl-1H-benzimidazole (2s, Table 2 , entry 19). White solid; m.p: $200-202{ }^{\circ} \mathrm{C}$ (EtOAc); ${ }^{1} \mathrm{H}$ NMR $\left(300 \mathrm{MHz}, \mathrm{CDCl}_{3}\right): \delta 2.17$ (s, 3H), 6.45 (s, 2H), $7.33-7.19(\mathrm{~m}, 4 \mathrm{H}), 7.43(\mathrm{t}, J=7.8 \mathrm{~Hz}, 1 \mathrm{H}), 7.68(\mathrm{~d}, J=8.7 \mathrm{~Hz}, 2 \mathrm{H}), 7.88(\mathrm{~d}, J=7.8$ $\mathrm{Hz}, 1 \mathrm{H}), 14.30(\mathrm{~s}, 1 \mathrm{H}) ;{ }^{13} \mathrm{C} \mathrm{NMR}\left(75 \mathrm{MHz}, \mathrm{CDCl}_{3}\right): \delta 14.5,47.5,111.7,111.9,119.6,120.3$, $122.7,123.9,124.3,124.4,134.0,135.5,142.0,143.3,143.5,143.9$; ESI-MS $(\mathrm{m} / z): 295.0$ $\left(\mathrm{M}^{+}+1\right)$; IR (KBr, cm$\left.)^{-1}\right): 767,1034,1241,1356,1453,2356,2907,2990,3040 \mathrm{~cm}^{-1}$; Anal. Calc. for $\mathrm{C}_{16} \mathrm{H}_{14} \mathrm{~N}_{4} \mathrm{~S}$ : C 65.28, H 4.79, N 19.03; Found: C 65.21, H 4.86, N 19.04.

Preparation of sulfoxides. In a round bottomed flask $(20 \mathrm{~mL})$, sulfide $(1 \mathrm{mmol}), \mathrm{NaBiO}_{3}(3$ $\mathrm{mmol})$, aqueous acetic acid $(4 \mathrm{~mL}, 50 \% \mathrm{v} / \mathrm{v})$ and acetone $(1 \mathrm{~mL})$ were mixed and refluxed for stipulated time (monitored by TLC). After the completion of the reaction, the reaction mixture was filtered through a pad of celite and diluted with water $(10 \mathrm{~mL})$. The mixture was extracted with EtOAc ( 3 x $10 \mathrm{~mL})$. The EtOAc extract was washed with sodium carbonate solution $(3 \mathrm{x} 10$ $\mathrm{mL})$, brine $(10 \mathrm{~mL})$, dried over anhydrous sodium sulfate and concentrated under reduced pressure. The crude product was separated by column chromatography using silica gel (60-120 mesh) and ethyl acetate-petroleum ether $\left(60-80{ }^{\circ} \mathrm{C}\right)$ as eluant. The characteristic data for the compounds are given below.

2-Methyl-1-methanesulphinylmethyl-1H-benzimidazole (3a, Table 3, entry 1). Pale yellow solid; m.p: $186-188{ }^{\circ} \mathrm{C}$ (MeOH and EtOAc); ${ }^{1} \mathrm{H}$ NMR (300 MHz, d6-DMSO): $\delta 2.56$ (s, 3H), $2.68(\mathrm{~s}, 3 \mathrm{H}), 5.44(\mathrm{~d}, J=13.8 \mathrm{~Hz}, 1 \mathrm{H}), 5.59(\mathrm{~d}, J=13.8 \mathrm{~Hz}, 1 \mathrm{H}), 7.20-7.15(\mathrm{~m}, 2 \mathrm{H}), 7.53-$ $7.50(\mathrm{~m}, 1 \mathrm{H}), 7.61-7.58(\mathrm{~m}, 1 \mathrm{H}) ;{ }^{13} \mathrm{C}$ NMR (75 MHz, $\mathrm{d}_{6}$-DMSO): $\delta 14.0,36.1,62.5,110.6$, 118.5, 122.0, 122.1, 135.3, 142.3, 153.1; ESI-MS (m/z): $209.0\left(\mathrm{M}^{+}+1\right)$; IR $\left(\mathrm{KBr}, \mathrm{cm}^{-1}\right): 856$, 1080, 1376, 1465, 1543, 2374, 2928, $2995 \mathrm{~cm}^{-1}$; Anal. Calc. for $\mathrm{C}_{10} \mathrm{H}_{12} \mathrm{~N}_{2} \mathrm{OS}$ : C 57.67, H 5.81 N 13.45; Found: C 57.58, H 5.88, N 13.47.

1-Methanesulphinylmethyl-2,5,6-trimethyl-1H-benzimidazole (3b, Table 3, entry 2). Pale yellow solid; m.p: $72-74{ }^{\circ} \mathrm{C}\left(\mathrm{MeOH}\right.$ and EtOAc); ${ }^{1} \mathrm{H}$ NMR (300 MHz, d6-DMSO): $\delta 2.24$ (s, 
3H), $2.26(\mathrm{~s}, 3 \mathrm{H}), 2.51(\mathrm{~s}, 3 \mathrm{H}), 2.71(\mathrm{~s}, 3 \mathrm{H}), 5.36(\mathrm{~d}, J=14.1 \mathrm{~Hz}, 1 \mathrm{H}), 5.52(\mathrm{~d}, J=14.1 \mathrm{~Hz}, 1 \mathrm{H})$, 7.28 (s, 2H), 7.37 (s, 1H); ${ }^{13} \mathrm{C}$ NMR (75 MHz, d6-DMSO): $\delta 14.0,19.9,20.18,36.1,62.7,110.8$, 118.7, 130.3, 130.5, 133.8, 140.8, 152.1; ESI-MS $(\mathrm{m} / \mathrm{z}): 237.0\left(\mathrm{M}^{+}+1\right)$; HRMS $(\mathrm{m} / \mathrm{z})$ for $\mathrm{C}_{12} \mathrm{H}_{16} \mathrm{~N}_{2} \mathrm{OS}$; calcd 236.0983, found 236.0973; IR $\left(\mathrm{KBr}, \mathrm{cm}^{-1}\right)$ : 846, 1050, 1398, 1465, 1527, 2371, 2919, $2974 \mathrm{~cm}^{-1}$; Anal. Calc. for $\mathrm{C}_{12} \mathrm{H}_{16} \mathrm{~N}_{2} \mathrm{OS}$ : C 60.99, H 6.82, N 11.85; Found: C 6O.87, H 6.91, N 11.88.

1-Methanesulphinylmethyl-2-phenyl-1H-benzimidazole (3c, Table 3, entry 3). Colorless crystalline solid; m.p: $68-70{ }^{\circ} \mathrm{C}\left(\mathrm{MeOH}\right.$ and EtOAc); ${ }^{1} \mathrm{H}$ NMR $\left(300 \mathrm{MHz}, \mathrm{CDCl}_{3}\right): \delta 2.42$ (s, $3 \mathrm{H}), 5.08(\mathrm{~d}, J=13.5 \mathrm{~Hz}, 1 \mathrm{H}), 5.19(\mathrm{~d}, J=13.5 \mathrm{~Hz}, 1 \mathrm{H}), 7.31-7.22(\mathrm{~m}, 2 \mathrm{H}), 7.50-7.47(\mathrm{~m}$, 4H), $7.77-7.69(\mathrm{~m}, 3 \mathrm{H}) ;{ }^{13} \mathrm{C}$ NMR $\left(75 \mathrm{MHz}, \mathrm{CDCl}_{3}\right): \delta 36.6,64.6,110.6,119.7,123.3,123.5$, 128.7, 129.8, 130.1, 135.1, 142.3, 153.7; ESI-MS (m/z): $271.0\left(\mathrm{M}^{+}+1\right)$; IR $\left(\mathrm{KBr}, \mathrm{cm}^{-1}\right): 753$, 1037, 1392, 1458, 2184, $3191 \mathrm{~cm}^{-1}$; Anal. Calc. for $\mathrm{C}_{15} \mathrm{H}_{14} \mathrm{~N}_{2} \mathrm{OS}$ : C 66.64, H 5.22, N 10.36; Found: C 66.56, H 5.26, N 10.40.

5-Methyl-1-methanesulphinylmethyl-2-phenyl-1H-benzimidazole (3d, Table 3, entry 4). Mixture of two tautomers; pale yellow solid; m.p: $68-70{ }^{\circ} \mathrm{C}\left(\mathrm{MeOH}\right.$ and EtOAc); ${ }^{1} \mathrm{H}$ NMR (300 MHz, $\left.\mathrm{CDCl}_{3}\right): \delta(2.46$ and 2.49) (two s, 3H, tautomeric), $2.51(\mathrm{~s}, 3 \mathrm{H}), 5.30-5.13(\mathrm{~m}, 2 \mathrm{H})$, (7.13 and 7.63) (two d, $J=8.1$ and $8.4 \mathrm{~Hz}, 2 \mathrm{H}$, tautomeric and aromatic), (7.34 and 7.53) (two s, $1 \mathrm{H}$, tautomeric and aromatic), $7.52-7.40(\mathrm{~m}, 3 \mathrm{H}), 7.80-7.70(\mathrm{~m}, 2 \mathrm{H}) ;{ }^{13} \mathrm{C} \mathrm{NMR}(75 \mathrm{MHz}$, $\left.\mathrm{CDCl}_{3}\right): \delta 21.4,21.8,36.9,64.9,110.5,110.7,119.3,119.3,125.4,125.5,128.2,128.5,129.0$, 129.0, 130.0, 130.0, 130.4, 130.6, 133.0, 133.9, 134.2, 135.3, 139.9, 141.7, 153.1, 153.5; ESIMS (m/z): $285.0\left(\mathrm{M}^{+}+1\right)$; IR (KBr, cm $\left.{ }^{-1}\right): 774,1082,1397,1478,1522,2284,2996 \mathrm{~cm}^{-1}$; Anal. Calc. for $\mathrm{C}_{16} \mathrm{H}_{16} \mathrm{~N}_{2} \mathrm{OS}$ : C 67.58, H 5.67, N 9.85; Found: C 67.49, H 5.72, N 9.89.

2-(4'-Chlorophenyl)-1-methanesulphinylmethyl-1H-benzimidazole (3e, Table 3, entry 5). Colorless crystalline solid; m.p: $94-96{ }^{\circ} \mathrm{C}\left(\mathrm{MeOH}\right.$ and EtOAc); ${ }^{1} \mathrm{H}$ NMR $\left(300 \mathrm{MHz}, \mathrm{CDCl}_{3}+\right.$ three drops of $\mathrm{d}_{6}$-DMSO): $\delta 2.49(\mathrm{~s}, 3 \mathrm{H}), 5.04(\mathrm{~d}, J=13.5 \mathrm{~Hz}, 1 \mathrm{H}), 5.15(\mathrm{~d}, J=13.5 \mathrm{~Hz}, 1 \mathrm{H})$, $7.27-7.19(\mathrm{~m}, 2 \mathrm{H}), 7.44-7.34(\mathrm{~m}, 3 \mathrm{H}), 7.71-7.66(\mathrm{~m}, 3 \mathrm{H}) ;{ }^{13} \mathrm{C} \mathrm{NMR}\left(75 \mathrm{MHz}, \mathrm{CDCl}_{3}+\right.$ three drops of $\mathrm{d}_{6}$-DMSO): $\delta 36.7,64.6,110.5,119.7,123.4,123.5,127.2,128.8,131.2,135.0$, 136.3, 142.2, 152.7; ESI-MS $(\mathrm{m} / \mathrm{z})$ : $305.0\left(\mathrm{M}^{+}+1\right)$; HRMS $(\mathrm{m} / \mathrm{z})$ for $\mathrm{C}_{15} \mathrm{H}_{13} \mathrm{ClN}_{2} \mathrm{OS}$; calcd 304.0437, found 304.0435; IR $\left(\mathrm{KBr}, \mathrm{cm}^{-1}\right)$ : 741, 1045, 1246, 1380, 1407, 1459, 1603, 2377 , 2919, 2996, $3054 \mathrm{~cm}^{-1}$; Anal. Calc. for $\mathrm{C}_{15} \mathrm{H}_{13} \mathrm{ClN}_{2} \mathrm{OS}$ : C 59.11, H 4.30, N 9.19; Found: C 59.02, H 4.37, N 9.21.

1-Methanesulphinylmethyl-2-(4'-nitrophenyl)-1H-benzimidazole (3f, Table 3, entry 6). Yellow solid; m.p: $212-214{ }^{\circ} \mathrm{C}$ (MeOH and EtOAc); ${ }^{1} \mathrm{H}$ NMR (300 MHz, d6-DMSO): $\delta 2.72$ $(\mathrm{s}, 3 \mathrm{H}), 5.50(\mathrm{~d}, J=14.1 \mathrm{~Hz}, 1 \mathrm{H}), 5.64(\mathrm{~d}, J=14.1 \mathrm{~Hz}, 1 \mathrm{H}), 7.38-7.30(\mathrm{~m}, 2 \mathrm{H}), 7.76(\mathrm{~d}, J=7.5$ $\mathrm{Hz}, 1 \mathrm{H}), 7.81(\mathrm{~d}, J=7.5 \mathrm{~Hz}, 1 \mathrm{H}), 8.20(\mathrm{~d}, J=8.4 \mathrm{~Hz}, 2 \mathrm{H}), 8.35(\mathrm{~d}, J=8.4 \mathrm{~Hz}, 2 \mathrm{H}) ;{ }^{13} \mathrm{C}$ NMR (75 MHz, $\mathrm{d}_{6}$-DMSO,): $\delta$ 36.3, 63.6, 112.1, 119.8, 123.4, 123.7, 123.8, 135.5, 135.8, 135.9, 142.5, 148.1, 152.0; ESI-MS (m/z): $316.3\left(\mathrm{M}^{+}+1\right)$; IR $\left(\mathrm{KBr}, \mathrm{cm}^{-1}\right): 746,857,1052,1343,1512$, 1599, 2448, $2922 \mathrm{~cm}^{-1}$; Anal. Calc. for $\mathrm{C}_{15} \mathrm{H}_{13} \mathrm{~N}_{3} \mathrm{O}_{3} \mathrm{~S}$ : C 57.13, H 4.16, N 13.33; Found: C 56.99, H 4.25, N 13.38 . 


\section{Acknowledgements}

One of the authors (PKT) thanks the University Grants Commission, New Delhi for his fellowship (SRF). Thanks are also to the CAS Instrumentation Facility, Department of Chemistry, University of Calcutta for spectral data. We thank EPSRC and the University of Reading for funds for the X-Calibur system. We also acknowledge grant received from UGC funded Major project, F. No. 37-398 / 2009 (SR) dated 11-01-2010.

\section{References}

1. Xi, N.; Huang, Q.; Liu, L. In Comprehensive Heterocyclic Chemistry; Katritzky, A. R.; Ramsden, C. A.; Scriven, E. F. V.; Taylor, R. J. K. Eds.; Elsevier: Oxford, 2008; Vol. 4, pp 143-364.

2. Marion, N.; Diez-Gonzalez, S.; Nolan, S. P. Angew. Chem, Int. Ed. 2007, 46, 2988.

3. Ionic Liquids in Synthesis; Wasserscheid, P.; Welton, T. Eds.; Wiley-VCH: Weinheim, 2003.

4. (a) Hermann, W. A. Angew. Chem., Int. Ed. 2002, 41, 1291. (b) Crudden, C. M.; Allen, D. P. Coord. Chem. Rev. 2004, 248, 2247.

5. (a) Burdon, M. G.; Moffatt, J. G. J. Am. Chem. Sc. 1966, 88, 5855. (b) Pfitzner, K. E.; Marino, J. P.; Olofson, R. A. ibid. 1965, 87, 4658. (c) Hayashi, Y.; Oda, R. J. Org. Chem. 1967, 32, 457.

6. (a) Gassman, P. G.; Amick, D. R. Tetrahedron Lett. 1974, 15, 889. (b) Foote, C. S.; Mazur, S.; Burns, P. A.; Lerdal, D. J. Am. Chem. Soc. 1973, 95, 588.

7. Shen, T. Y.; Matzuk, A. R.; Dorn, C. P., Jr. (Merck and Co., Inc.). U.S. 1970, U.S. 3438 992.

8. (a) Janzen, A. F.; Lypka, G. N.; Wasylishen, R. E. Can. J. Chem. 1980, 58, 60. (b) Janzen, A. F.; Lypka, G. N.; Wasylishen, R. E. J. Heterocycl. Chem. 1979, 16, 415.

9. Frenanez, I.; Khiar, N. Chem. Rev. 2003, 103, 3651.

10. Kaczorowska, K.; Kolarska, Z.; Mitka, K.; Kowalski, P. Tetrahedron 2005, 61, 8315 and references therein.

11. Kirihara, M.; Yamamoto, J.; Noguchi, T.; Hirai, Y. Tetrahedron Lett. 2009, 50, 1180 and references therein.

12. Rostami, A.; Akradi, J. Tetrahedron Lett. 2010, 51, 3501.

13. Banik, B. K.; Fernandez, M.; Alvarez, C. Synth. Commun. 2005, 35, 3065.

14. Kon, E.; McNeils, E. J. Org. Chem. 1976, 41, 1646. 